\title{
Analysis of the Electric Field Distribution within MV Cable Joint in the Presence of Defects in Cable Insulation
}

\author{
Sobhy S. Desouky \\ Electrical Engineering Dept. \\ Faculty of Engineering, \\ Port-Said University, \\ Port-Said, Egypt \\ sobhyserry@yahoo.com
}

\author{
Adel Z. El-Dein \\ Electrical Engineering Dept., \\ Faculty of Energy Engineering, \\ Aswan University, \\ Aswan, Egypt \\ azeinm2001@ hotmail.com

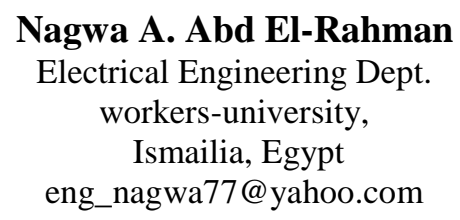

\author{
R.A.Abd El-Aal \\ Electrical Engineering Dept. \\ Faculty of Engineering, \\ Port-Said University, \\ Port-Said, Egypt \\ ramadanhv@gmail.com
}

Abstract - Power cables have a great importance in power transmission and distribution systems. Joints are the main accessories of the power cables. They are necessary to make connections between lines. The design of a cable joint mostly depends on the cable type, the applied voltage and the cores. These factors add to the way of how electric field stress is distributed at the cable joint. This paper presents a numerical analysis study to the stress control layering electric field distribution within a cable joint. In this paper, a 2D Finite Element Method (FEM) is used.

A developed program using FEM has been used to simulate the electric field distribution in the joint of a single core crosslinked polyethylene (XLPE) underground medium voltage cable, as well as to investigate the presence of the defects and water droplets in the joint.

Many factors such as: the defect size and location, insulation material dielectric constant, insulation thickness, as well as the cavity dimensions, shapes (cylindrical or spherical) and number (one to three either in horizontal or in vertical formation) have been investigated. Also, the electric field in water droplet having different shapes (spherical, hemispherical and cylindrical) is presented. The electric field at this region is increases the electrical discharges at the defect sites. In turn the breakdown occurs when the magnitude of the electric field is larger than the breakdown strength. The results obtained assist in the design of cable joint structures, which can increase the reliability of cable system.

Keywords-cable joint, defects, electric field, finite element method, COMSOL metaphysics.

\section{INTRODUCTION}

Power cables have many shapes, sizes, and configurations [1].

Variety of considerations have to be taken into account while designing the cable terminations and joints because they must have the same reliability as their related cables while making the connection for both indoor and outdoor applications [2].

Cable joint is used to connect two cables together. The conductors are usually connected together with a connector that can be welded, pressed or tightened by a screw [3].

The surface of a connector should be cylindrical and smooth to improve the electric field in the joint and avoid the generation of electric field peaks [3]. In addition, the connector has to be smoothed by applying a semiconductor layer on its top. It is necessary to use a connector with big radii at both ends in order to make sure that the peaks in the electric field don't compromise the insulation.

The material of insulation of the joint depends on the kind of used cables [4].

Shielded power cables require electric field stress control when they are terminated because the high potential gradients are concentrated in the cutback point causing high electric stress when the insulation shield is removed from a cable.The improvement of the electrical field at these points can cause local discharges, which could give either flashover along the insulation surface or electrical breakdown causing cable failure [5]. Cable joint is the weakest point in the transmission line because its defects may cause a system failure. These defects exist due to poor installation, manufacturing process or during in-service. The partial discharge is the general cause of the failure in the cable joint and this is the main reason for system breakdown [6]. There are some kinds of defects in the cable joint such as cavity, insulator tip, insulation cut etc.

Parameters Affecting the Joints:

a. The applied voltage: Cable joints exist for low, medium and high voltage power cables. The joints always have a

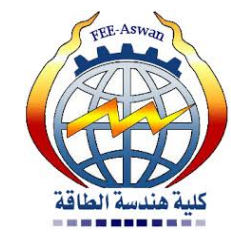


lower power capacity than the cable itself, and the joints may fail under the stress of the electrical current.

b. The structure: the joints of cables have different basic constructions based on the connection of cables. Some cable joints are simply connected to power cables at a single point, while others are placedin the main line.

C. The material of insulation: Differentkinds of cable joints are installed in different kinds of power cables. Power cables can include layers of polymeric insulation such as cross-linked polyethylene (XLPE), polyethylene (PE) and ethylene propylene rubber (EPR) and require cable joint, which is compatible with the cable dielectric (insulation) [7].

More than $60-70 \%$ of failures in medium voltage (MV) cable networks are due to internal defects in the cable insulation system [8].

The distribution of the electric field inside and around the insulating structures is an important consideration which should be taken into account by the engineers who design the tools that can be operated at high voltage levels [9]. The highest stress in the insulating system is the key factor that should be controlled.

The distribution of the electric field within the dielectric changes and distorts because of the existence of defects. The possibility of failure increases if cavities are shaped during the production process of insulation or during the operation of a high voltage system [10].

\section{FINITE ELEMENT ANALYSIS OF MV CABLE JOINT}

The goal of this analysis software is to get appropriate numerical formulation of the specified non-linear partial differential equations to calculate the electric potential. Therefore, the original problem changes with the different types of equations used in the finite element model. The finite dimension is presented by the complete basis set and the repeated orders are performed to get the ideal solution [11].

In highly non-homogeneous fields, this assumption may not give precise results. The accuracy of calculations can be easily improved by making the net of the element denser. So, the number of calculations increases and cannot be done unlimitedly [12].

COMSOL program is a simulation software that uses the finite element method. The key idea of FEM is to divide the area under study into small elements, where every field's magnitude is obtained by a function.

The voltage $(V)$ might be used as field magnitude when the static electric fields under study minimize the interfaces from heat transfer and fluid flow to structural mechanics and electromagnetic study [13].

\section{FINITE ELEMENT COMPUTATIONS}

Finite element calculations have been taken in the twodimension(2D) axial symmetric model of the geometry of a medium-voltage cable joint.In addition, they have been introduced using the FEA software [6] as shown in Fig.1. The distribution of electric field in the structure of the MV cable joint is simulated using this model.

Table I shows the dielectric constants of different layers in the cable joint model [7]. The applied voltage of the conductor is $20 \mathrm{KV}$, the semiconducting layer is grounded and the ferrule electrode is assumed to be a floating potential.

Table II shows the values of all constants and variables used in the computation process.

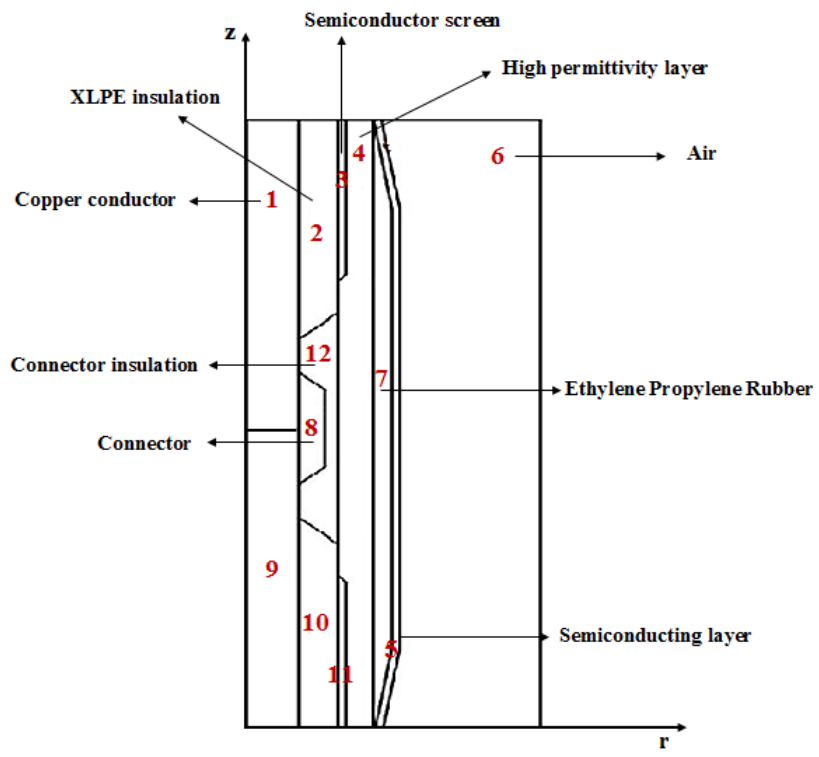

Fig. 1. Geometry of (2D) MV cable joint model

TABLE I. DIELECTRIC CONSTANT OF MV CABLE JOINT MODEL

\begin{tabular}{|l|l|}
\hline \multicolumn{1}{|c|}{ Layers } & \multicolumn{1}{c|}{ Dielectric Constant,-.. } \\
\hline XLPE insulation (2,10) & 2.3 \\
\hline Copper conductor (1,9) & 1 \\
\hline High permittivity layer (4) & 25 \\
\hline Semiconductor screen (3,11) & 2.26 \\
\hline Semiconducting layer (5) & 100 \\
\hline Ethylene Propylene Rubber (7) & 3.4 \\
\hline Connector (8) & 1 \\
\hline Connector insulation (12) & 50 \\
\hline Air (6) & 1 \\
\hline
\end{tabular}

TABLE II. VALUES OF CONSTANTS AND VARAIABLES USED IN COMPUTATION PROCESS

\begin{tabular}{|l|l|}
\hline \multicolumn{1}{|c|}{ Parameter } & \multicolumn{1}{c|}{ Value } \\
\hline Applied voltage of the conductor & $20 \mathrm{KV}$ \\
\hline Connector insulation dielectric constant & $10,50,100$ \\
\hline HPL dielectric constant & $20,25,40$ \\
\hline EPR dielectric constant & $2,3.4,5$ \\
\hline Semi conducting dielectric constant & $80,100,120$ \\
\hline EPR thikness & $4,6,8 \mathrm{~mm}$ \\
\hline Connector insulatio thikness & $8 \mathrm{~mm}$ \\
\hline HPL thikness & $5.6 \mathrm{~mm}$ \\
\hline
\end{tabular}

\section{SIMULATION RESULTS}

The results of the medium voltage cable consist of electric field distribution by using COMSOL program in simulation model with and without defects. The simulation is done to study the effects of the different relative permittivity in MV layers as in high permittivity layer (HPL),in ethylene propylene rubber (EPR) layer, and in semi conducting layer. The obtained results present the calculation of electric field distribution in MV cable joint, with and without cavities of cylindrical or spherical shapes and various numbers (from one to three either in horizontal or in vertical formation). Also, the obtained results present the calculation of electric field distribution with and without water droplet inside the EPR layer. Where, the electric field distribution at spherical 
water, hemispherical, and cylindrical shapes are simulated and presented.

\section{A. The Distributionof the Electric field and the Electric Potential}

Figs. 2 and 3 show the distribution of the electric potential and the electric field within and around the medium-voltage joint. From these figures, it is noticed that the electric field and the equipotential distribution in the MV cable joint are simulated by two-dimensional field models. The electric potential is higher on the conductor and electrode layers. However, it decreases in the outer layer of the cable joint which is grounded. As a result, this causes the electric field distribution to be graduated between the conductor and the electrode through the insulation material.

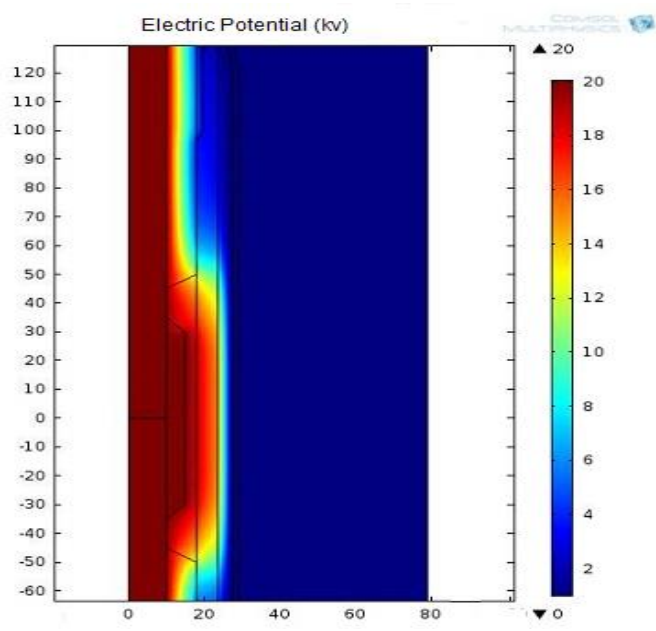

Fig. 2. Electric potential distribution in the MV cable joint

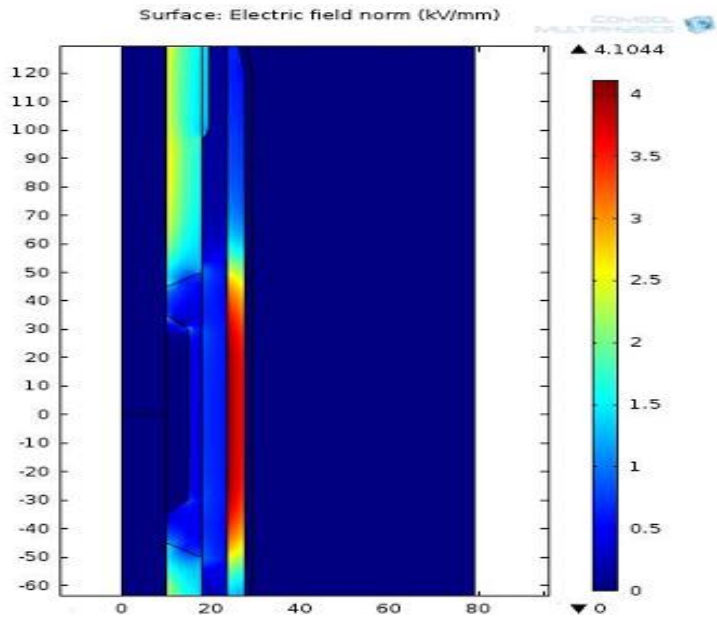

Fig. 3. Distribution of the electric fieldin the MV cable joint

\section{B. The influence of Different dielectric constantson $M V$ Cable Joint}

Figs. 4. -7. show the impact of the different dielectric constants on the medium cable joint layers as in the HPL layer, semiconductor layer, EPR layer, and ferrule insulation layer. The dielectric constant of the "connector" affects significantly the distribution of the electric field.

Fig. 4. presents the calculated electric field along the MV joint with different dielectric constants of the connector (insulation): 10, 50, and 100. The low dielectric constant results in increasing the concentration of the high electric field in the ferrule "connector" insulation, while the high dielectric constant results in increasing the electric field in the EPR layer as shown by in Fig. 4.

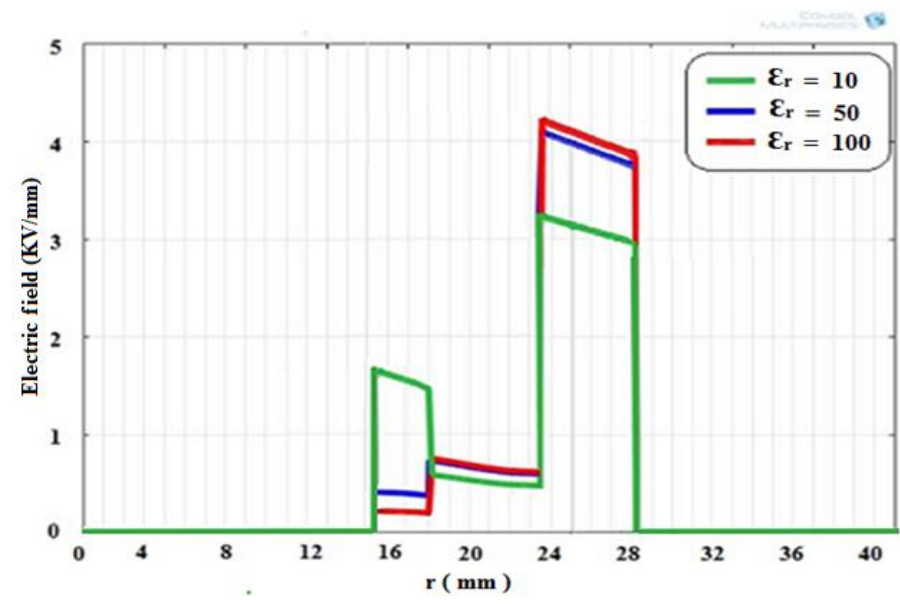

Fig.4. Variation of the electric field in the MV cable joint for different connector insulation dielectric constants

Fig. 5. shows that the distribution of the electric field in the HPL decreases when the dielectric constant of the high permittivity layer increases, while the electric field in other regions increases. In Fig. 6. it is noticed that when the dielectric constant of the EPR layer increases, the magnitude of the electric field in the ferrule insulation, HPL, and semiconductor layer increases. However, it decreases in the EPR layer. This means that the higher dielectric constant of HPL and EPR layer makes the electric field in other areashigher but it lowers the electric field inside these materials.

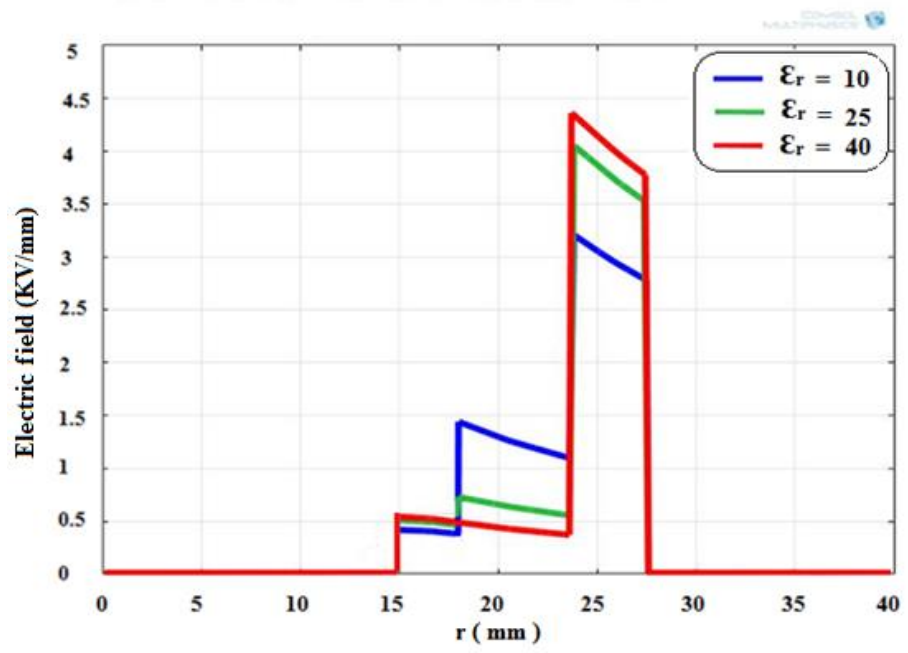

Fig. 5. Variation of the electric field in the MV cable joint for different HPL dielectric constants

Fig.6. indicates that the increase in the dielectric constant of the EPR layer with 2, 3.4, and 5 results in decreasing the electric field in EPR layer. The use of the highest dielectric constantthat equals 5 results inapproximately $13 \%$ decrease in the electric field peak compared to the dielectric constant which equals 3.4 (references case).

On the other hand, with the dielectric constant that equals2, the magnitude of the electric field increases by about $20 \%$ compared to the one that equals 3.4 but at the 
same time, it increases the electric field in the connector and the HPL layer.

Fig. 7. presents the effect of the variation in the dielectric constants on of the semiconducting layer. on the calculated electric field. It is noticed that the change in the dielectric constant of the semiconducting layer has no impact on the electric field magnitude in the parts of cable joint.

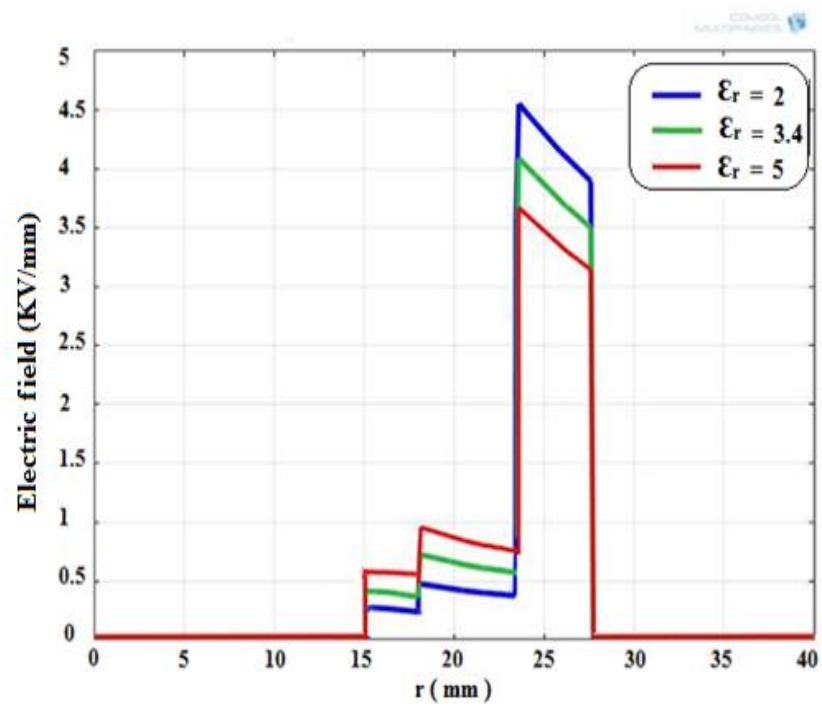

Fig. 6. Variation of the electric field in the MV cable joint for different EPR layer dielectric constants

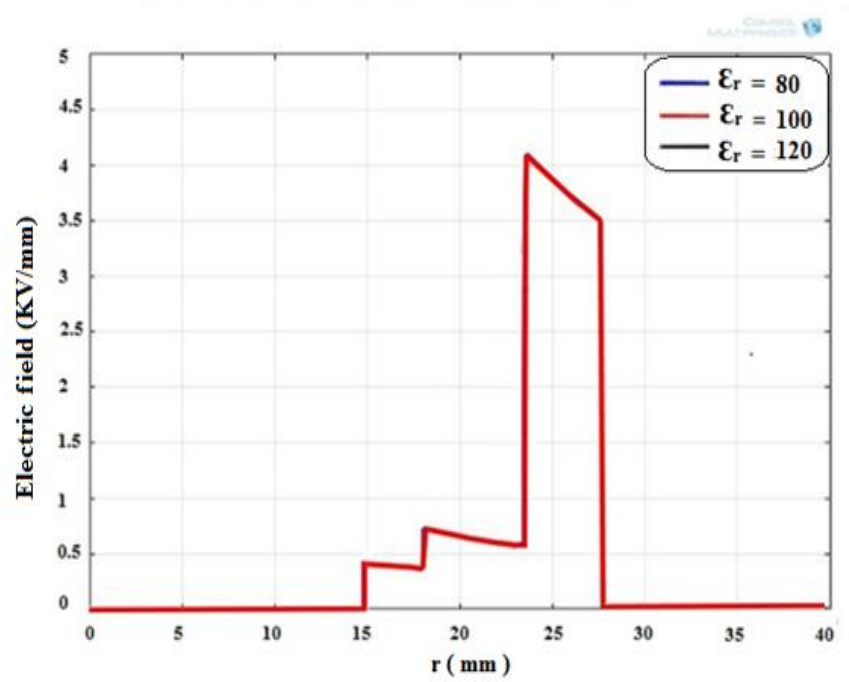

Fig. 7. Variation of the electric field in the MV cable joint for different semi coductor layer dielectric constants

\section{The Influence of Insulation Thickness}

In this part of our study, the simulation presents the effect of changing the thickness of the EPR layer on the distribution of the electric field in MV joint. The simulation is performed with three different levels of thickness $(4 \mathrm{~mm}$, $6 \mathrm{~mm}$, and $8 \mathrm{~mm})$

From Fig. 8. it is noticed that the electric field decreases when the thickness of the EPR layer increases. Therefore, there is a reverse effect between the layer thickness and the maximum electric field. In addition, the shape of the electric field becomes wider as the EPR layer thickness increases.

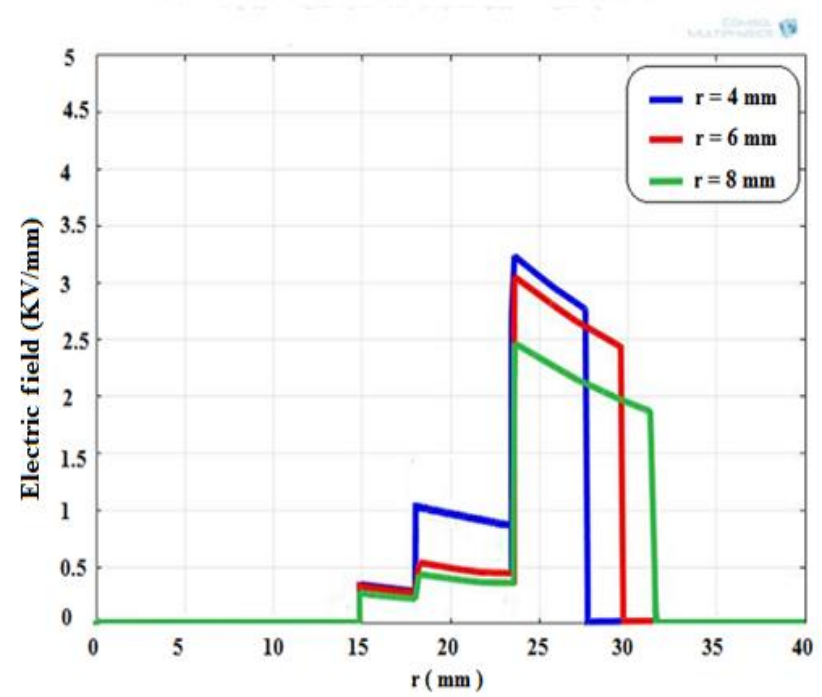

Fig. 8. Variation of the electric field in the MV cable joint for different EPR layer thiknesses

\section{The Influence of shape and location of cavity}

Defects in the EPR layer could be caused bythe manufacturing setting or operation and such cavities result in increasing the electric field causing the breakdown.

Figs. 9. -10. present the layer/ cavity of spherical cavity of radius $(r)$, located in EPR layer with thickness $(t)$ and the cylindrical cavity with diameter $(b)$ and height $(a)$, respectively.

The cavity is supposed to be filled with air. Due to the difference of the dielectric constant, the field improvement will take place in the cavity. 


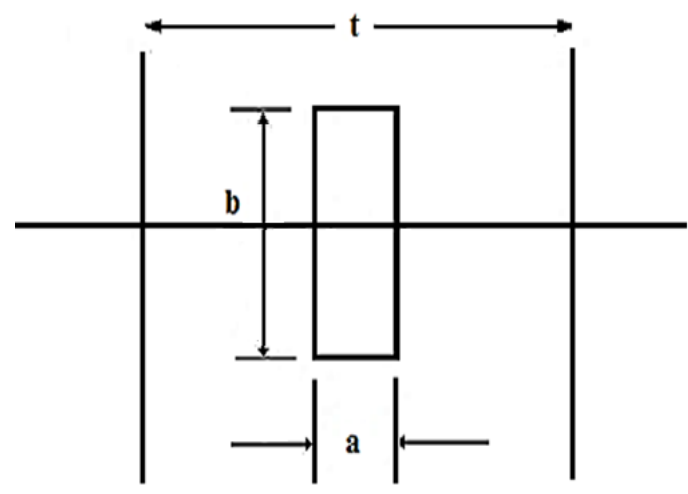

Fig. 10. (2-D) cylindrical cavity model

Figs.11. -12. show the impact of air cavity shape on the electrical field distribution in the HPL and the EPR layer.

The simulation indicates that the magnitude of the electric field increases in the presence of cavities. Fig.11. shows that the electrical field increases in the area of HPL, but Fig. 12. indicates that the increase takes place in the area of EPR layer with cavities. In addition, it is noticed that the spherical cavity of the size $2 r / t$, which is located in the center of the layer has the greatest effect on the maximum stress.

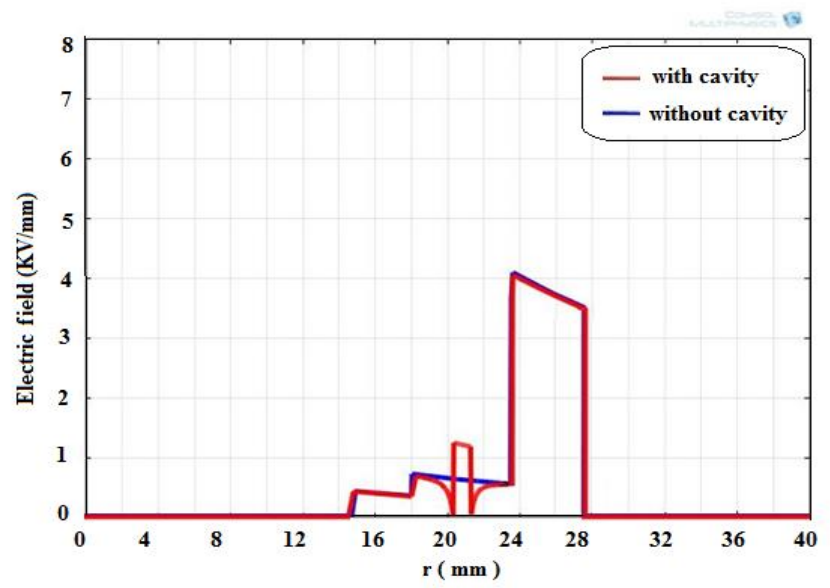

Fig. 11.Variation of the electric field in the MV cable joint with and without cavity in the HPL

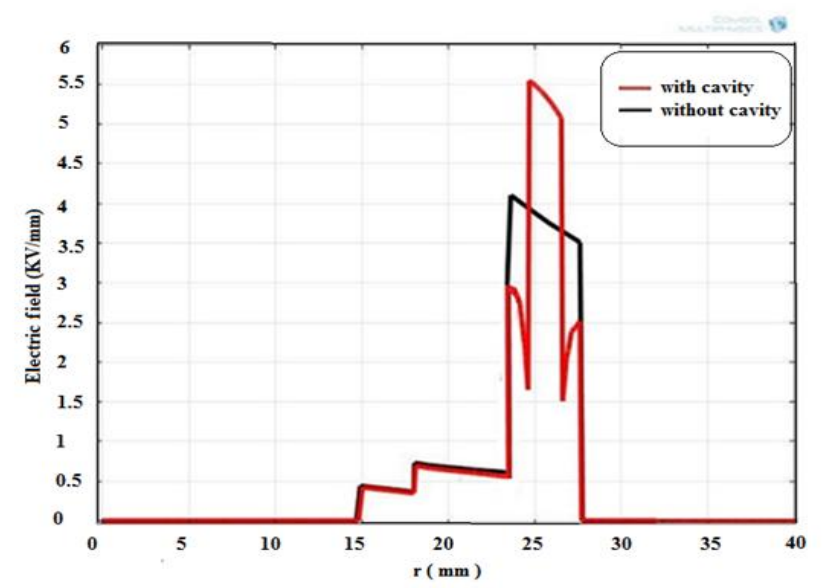

Fig.12.Variation of the electric field in the MV cable joint with and without cavity in the EPR layer
Fig. 13. shows that when the ratio $2 r / t$ increases, the electric field decreases due to the increase in the volumetric charge in the cavity, which reduces the applied field.

It is noticed that there is no increase in the electric field when the ratio $2 r / t$ reaches 1 because this makes an air gap between the two layers.

Figs 14. - 16. show the impact of different number of horizontal cavities within the EPR layer. For constant $(2 r / t)$, it is noticed that the electric field of a single cavity, which is located in the center, is higher than that in the cases of twoand three-series cavities. This is because the ionization in the first cavity will push the electrons to move in one direction and the positive ions to move in the opposite direction. Therefore, this may introduce a reverse field in the EPR layer from the second to the first cavity and accordingly the magnitude of the electric field will decrease. The situation will be greater for the three cavities in the horizontal position.

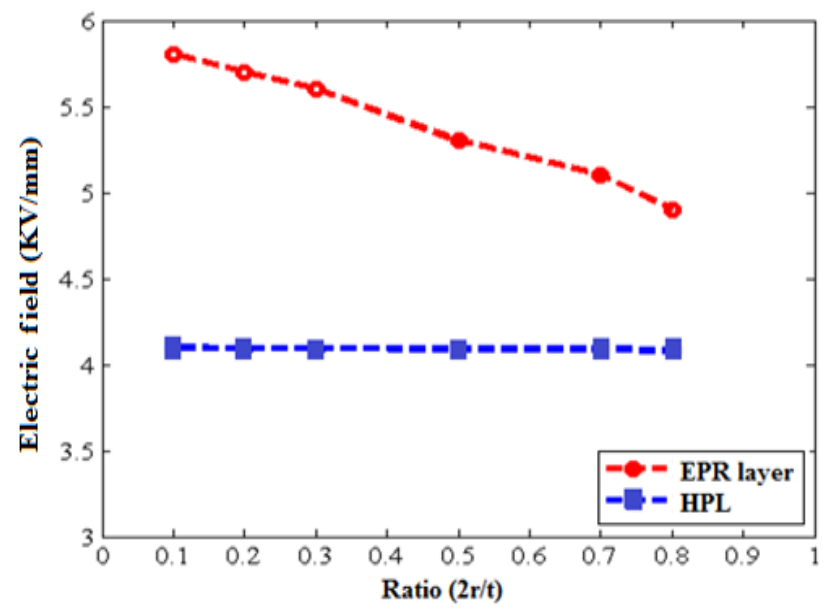

Fig. 13. Variation of the electric field in spharical cavity located at the center of EPR layer

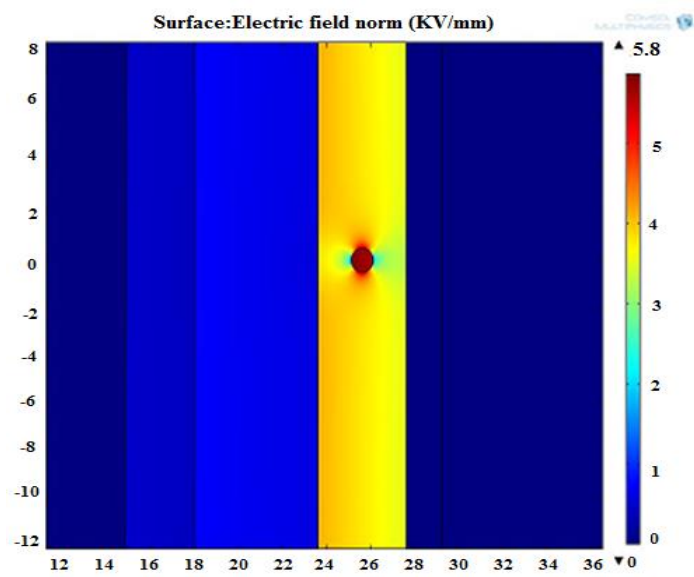

Fig. 14. Distribution of the electric field in MV cable joint with one center horizontial cavity in the EPR layer $(2 r / t=0.2)$ 


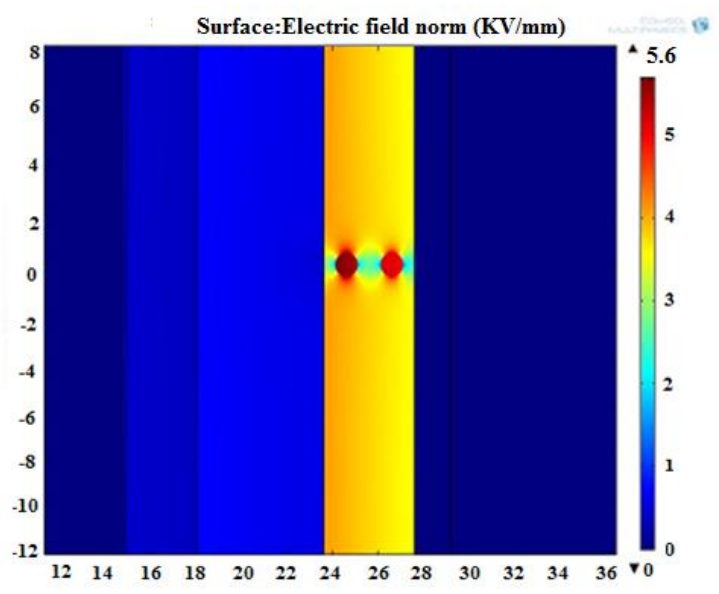

Fig. 15. Distribution of the electric field in MV cable joint with two horizontial cavity in the EPR layer $(2 r / t=0.2)$

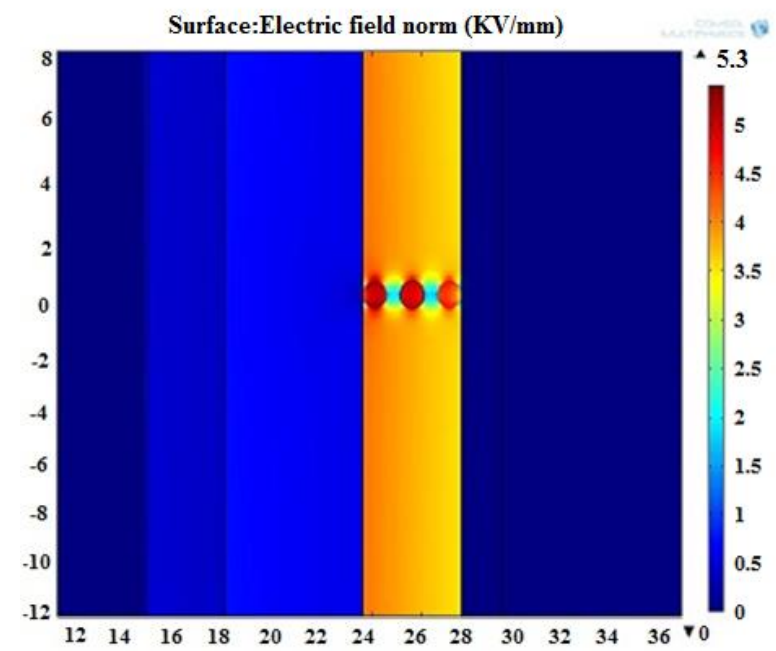

Fig. 16. Distribution of the electric field in MV cable joint with three horizontial cavity in the EPR layer $(2 r / t=0.2)$

Based on Figs 17. - 18., it is noticed that for constant value of $2 r / t$, the magnitude of the electric field in the configuration of the three vertical cavities is higher than that in the configuration of the two vertical cavities.

In case of the configuration of one cavity, the magnitude of the maximum electric field is the least one. In addition, based on the results, which have been found, it is noticed that the electric field is higher in the vertical case than the one in the horizontal case. This is due to the increase in the volumetric charge which causes the partial discharge within cavities and causes the damage of the dielectric as a result.

For cylindrical cavities with the same applied voltage, it is obvious that when the number of series of cavities increases, the maximum electric field in a given cavity increases.

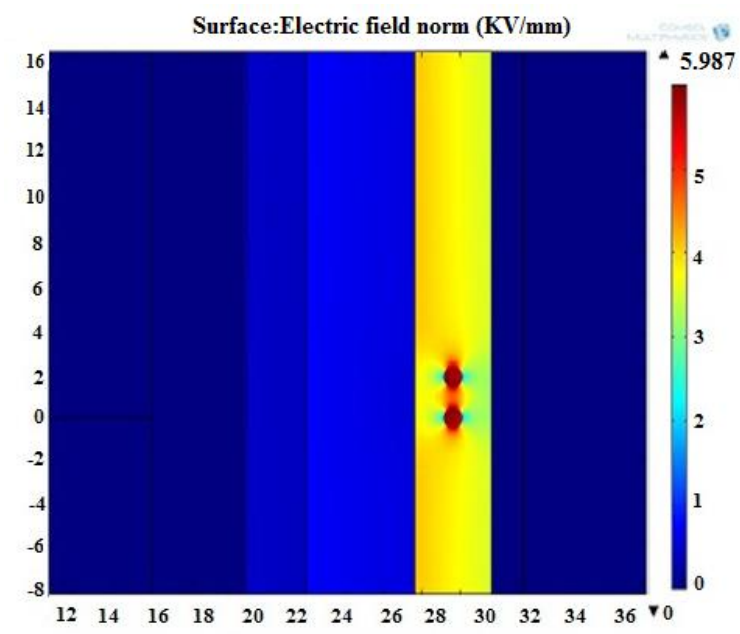

Fig. 17. Distribution of the electric field in MV cable joint with two vertical cavity in the EPR layer $(2 r / t=0.2)$

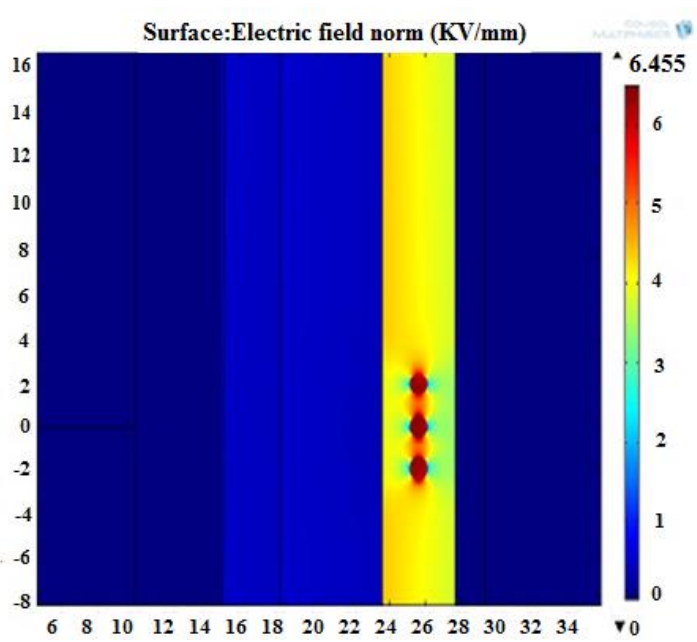

Fig. 18. Distribution of the electric field in MV cable joint with three vertical cavity in the EPR layer $(2 r / t=0.2)$

For a given cavity thickness ratio $(\mathrm{a} / \mathrm{t})$, the maximum electric field increases as the ratio (b/a) increases, i.e. the cavity diameter increases for given (t) and (a) values. This is due to the fact that the expected area increases compared to the used voltage. The possibility of the discharge is larger for the three series of cavities than that of the one or two series of cavities. Therefore, the breakdown increases when the discharge starts in any of the cavities. Consequently, the increase in the volume of series of vertical cavities will cause an increase in the electric field in every cavity and the possibility of breakdown will increase because the solid material separating the layers decreases.

Figs. 19. - 21. shows the simulation results of one, two, and three cylindrical cavities which are located in the EPR layer. 


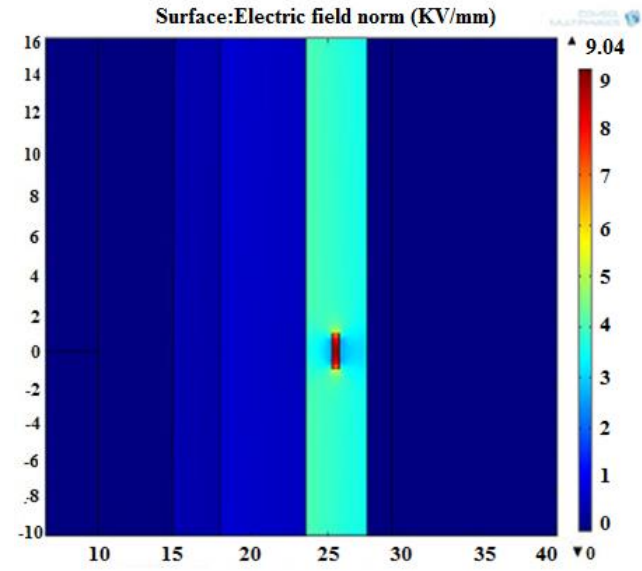

Fig. 19. Distribution of the electric field in MV cable joint with one vertical cylindrical cavity in the EPR layer $(b / a=3)$

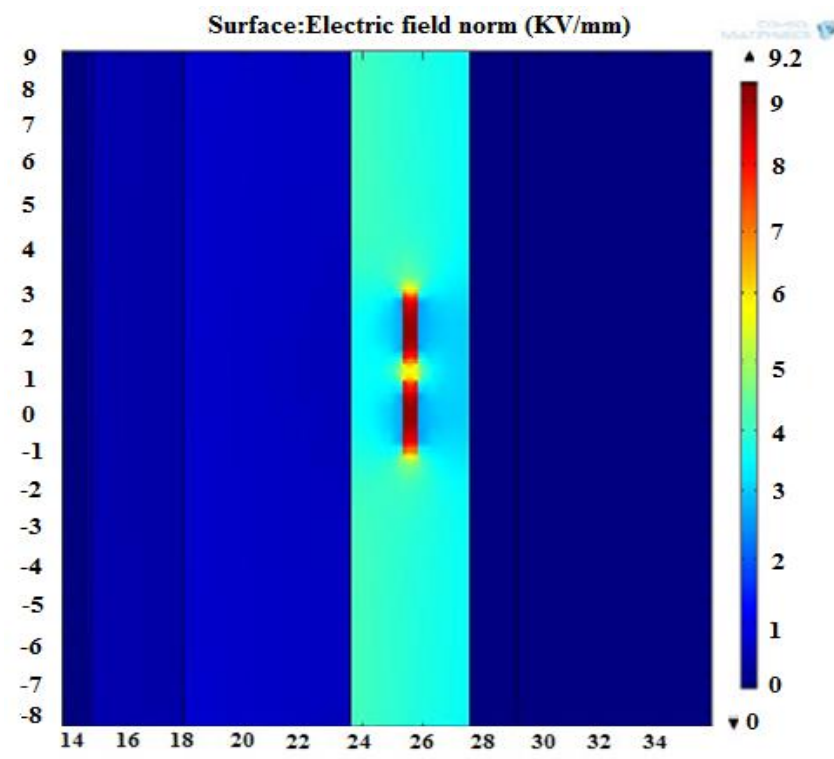

Fig.20.Distribution of the electric field in MV cable joint with two vertical cylindrical cavity in the EPR layer $(b / a=3)$

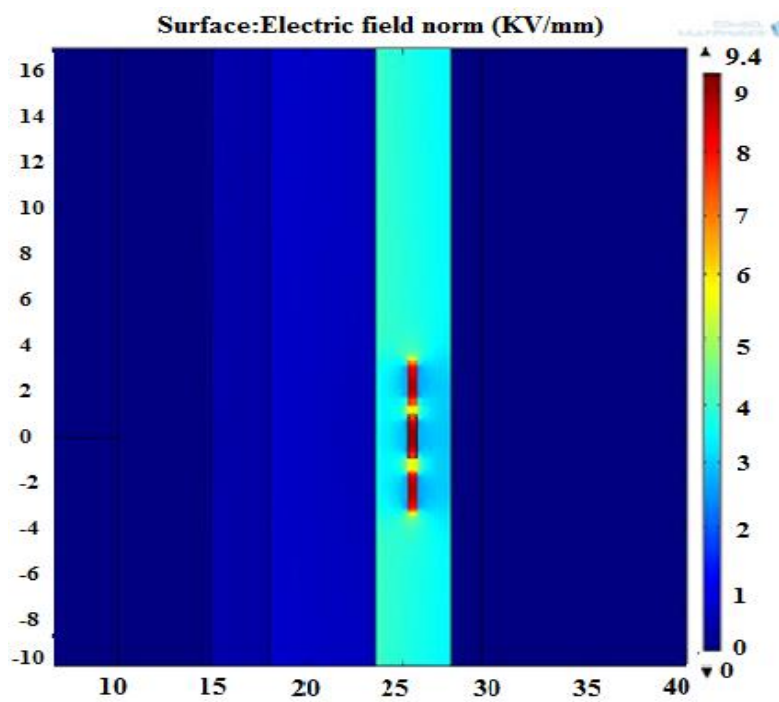

Fig.21.Distribution of the electric field in MV cable joint with three vertical cylindrical cavity in the EPR layer $(b / a=3)$

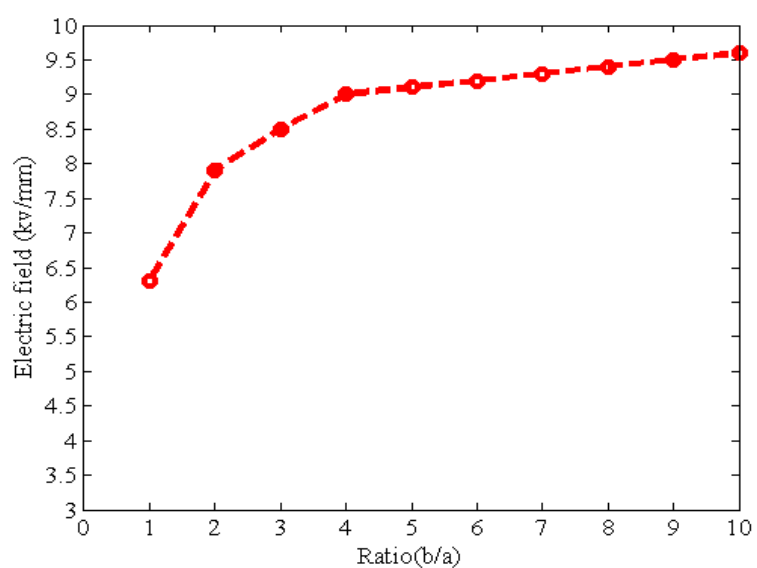

Fig.22. Variation of the electric field in vertical cylindrical cavity at the center of EPR layer $(a / t=0.125)$

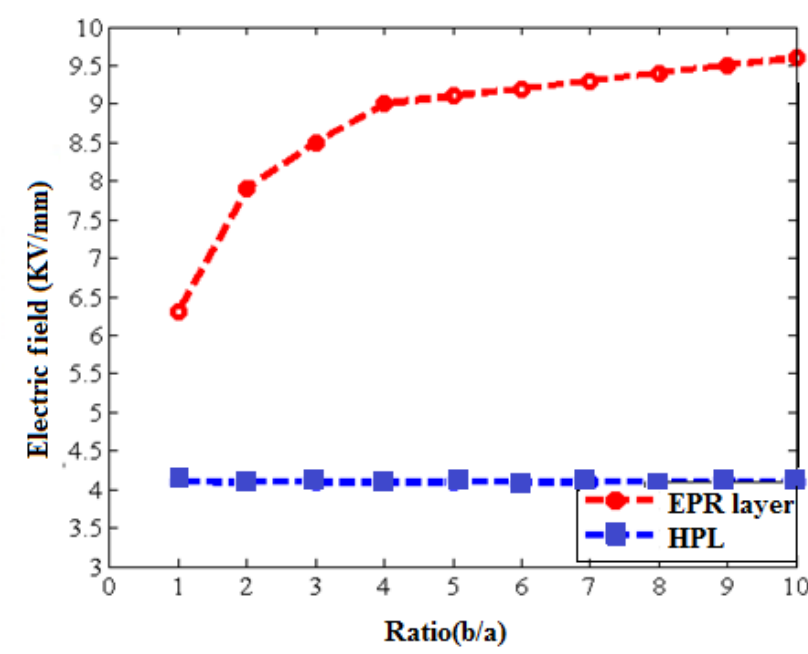

Fig. 23.Variation of the electric field in vertical cylindrical cavity at the center of HPL an EPR layer $(a / t=0.125)$

For the dielectric constant in the EPR layer; $\varepsilon_{r}=3.4$, with one cavity in the cylindrical shape located at the center of the EPR layer, the size of cavity has remarkable effects on the maximum electric field as shown in Figs. 22. - 23. It is noticed that the reliance of the maximum field on the cavity size is represented by the $(a / t)$ ratio. The results show that for a given ratio of cavity diameter to thickness ratio $(b / a)$, the maximum electric field increases as the ratio $(b / a)$ increases or as the cavity diameter increases for the given values of $t$ and $a$. This is due to the increase in the expected area to the used voltage. It may be noticed that from Figs. 18. - 21. the cylindrical-shaped cavities are much more vulnerable to partial discharge compared to the spherical shaped cavity.

\section{E. Effect of water droplets}

Water droplets in the EPR layer may be formed by condensation of gases because of the heat generated in the cable joint. Water droplets might make an excessive electric field which can cause the breakdown.

Droplets have different shapes; (hemispherical, spherical, deformed and cylindrical water drops). The results indicate that, the electrical field is uniformity distributed in the case 
without droplet but in the cases with droplets, the electrical field is not uniformity distributed.

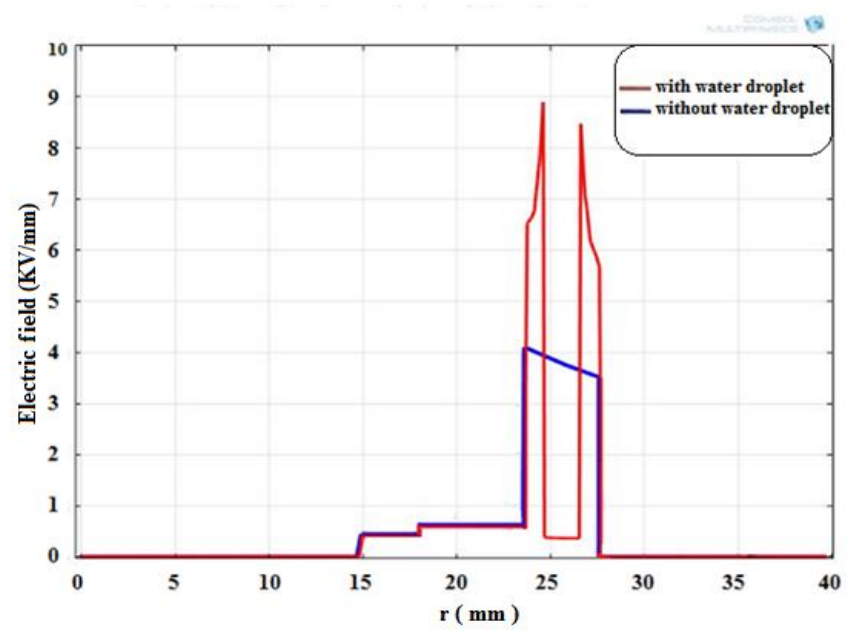

Fig. 24. Variation of the electric field in MV cable joint with and without water droplets on the EPR layer

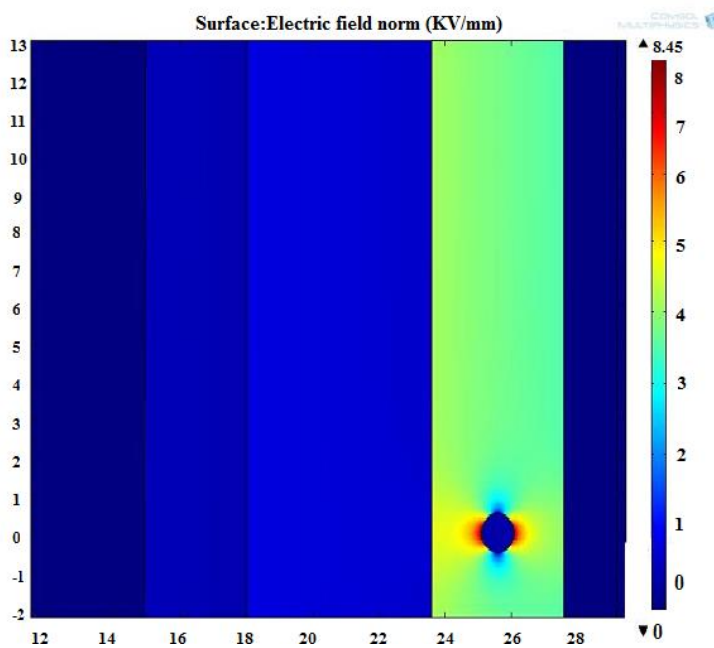

Fig.25.Distribution of the electric field in MV cable joint with spherical droplets on the EPR layer

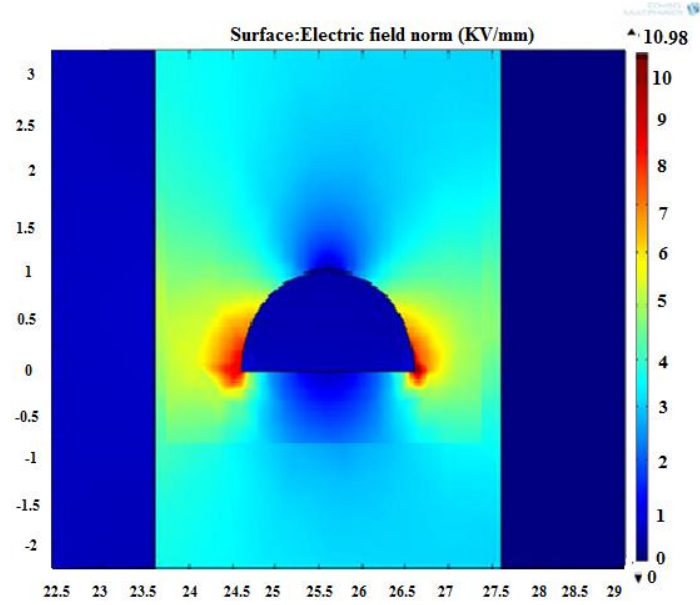

Fig.26.Distribution of the electric field in MV cable joint with hemispherical droplets on the EPR layer

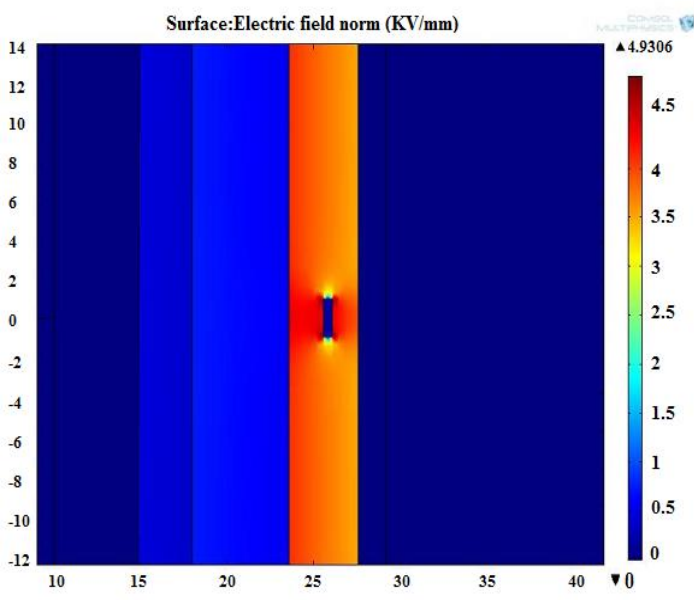

Fig.27.Distribution of the electric field in MV cable joint with cylindrical droplets on the EPR layer

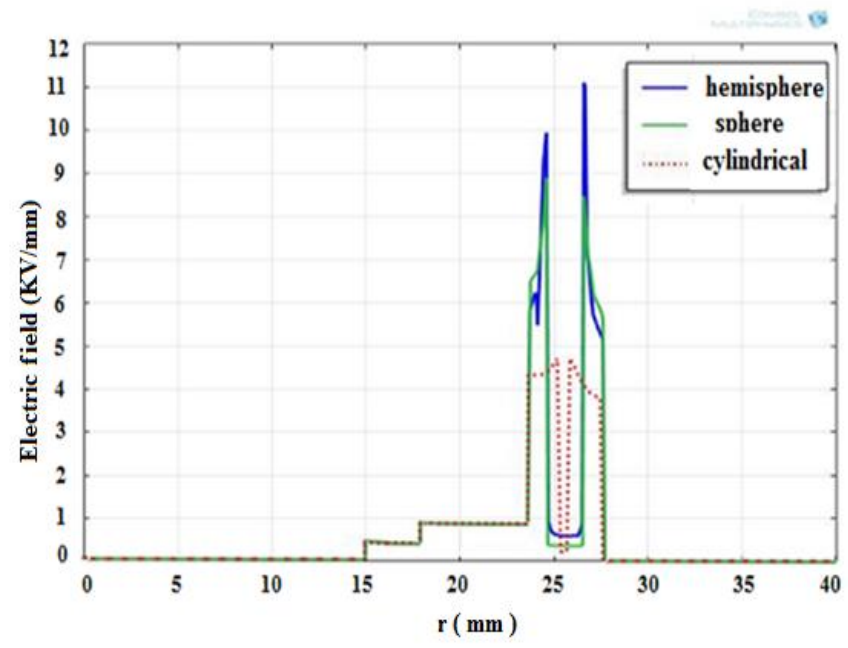

Fig. 28. Electric field in EPR with spherical, hemispheric, and cylindrical droplets

Fig. 24. shows the variation of the electric field in the EPR layer with and without water droplets. It may be remarked that the total electric field increases as a result of water droplets. In addition, It may be remarked that the electric field in the water droplet found on the joint structure is less than that in the surrounding insulation. This is because the dielectric constant of water droplet has a higher value equal around 80 times that of the surrounding insulation. The electric field distribution in the hemispheric, cylindrical and spherical droplets is shown in Figs. 25. - 27.

Fig.28. shows the distribution of the electric field with different droplet shapes (spherical, hemispherical, and cylindrical).The figure illustrates that the highest electric field exists in the hemispherical case compared to the spherical one. Compared to the case without water droplets, the water droplets intensify the local electric field stress.

This intensification is strongly dependent on the geometrical configuration of the droplet which is deformed once again by the alternating electric field.

\section{CONCLUSION}

The results of simulation of the MV cable joint indicate that the distribution of the electric field depends on the dielectric constant, the thickness of layers, and the existence of defects (air cavity, water droplets). 
The effect of varying the dielectric constant on the electric field magnitude in different layers is studied, it is concluded that the electric field increases with the increase of the dielectric constant of HPL and the connector. On the other hand, increasing the permittivity of EPR layer decreases the electric field in EPR layer but the electric field in the connector and in HPL increases, and increasing the thickness of EPR layer or increasing the relative permittivity reduces the total electric field. The change in the relative permittivity in semiconducting layer doesn't affect the electric field.

The electric field increases with the presence of cavity and water droplet which gives higher electric field than that with the air void. The study computes the distribution of electric field in spherical and cylindrical air voids as follow:

For spherical cavities, it was noticed that as the ratio of $(2 r / t)$ is increased, the maximum electric field is decreased, vertical voids configuration is exposed to higher electric field than horizontal ones in EPR layer.

For cylindrical cavities, the electric field increases as $b / a$ ratio increases at constant $a / t$ ratio and the stress increases by increasing the number of series cylindrical cavities, for the vertical configuration.

Also, it is concluded that, cylindrical voids (cavities) are much more vulnerable to partial discharge than spherical voids (cavities); This may be due to the increased projected area to the applied voltage.

The study shows also the effects of water droplets with spherical, hemispherical, and cylindrical shapes.

The obtained results indicate that the electric field increases in the case of hemispherical shape than that of other cases.

\section{REFERENCES}

[1] M. S. Naidu, and V. Kamaraju, "High voltage engineering", $2^{\text {nd }}$ ed., McGraw-Hill, 1996.

[2] G. Bas, "Electric field analysis in stress controlled high voltage cables", M.Sc.Thesis, Middle East Technical University, 2005.
[3] K. Vakevainen, "The effect of material properties to electric field distribution in medium voltage underground cable accessories", M.Sc.Thesis, Arcada University, Finland, 2010.

[4] S.V.Nikolajevic, N.M.Pekaric-Nad, and R.M.Dimitrijevic, "The influence of some construction parameters on electric stress grading in xlpe cable terminations", 2-5 June 1997, Conference Publication No.438, CIRED.

[5] H. A. Illias, Z. H. Lee, A. H. A. Bakar,and H. Mokhlis, "Distribution of electric field in medium voltage cable joint geometry", IEEE International Conference on Condition Monitoring and Diagnosis 2327 September 2012, Bali, Indonesia.

[6] E. Kuffel, W. S. Zaengl, and J. Kuffel, "High voltage engineering: fundamentals", $2^{\text {nd }}$ ed, Butterworth-Heinemann, Newnes, 2000.

[7] X. Wang, C.C. Wang, K. Wu, D.M. Tu, S. Liu, and J.K. Peng, "An improved optimal design scheme for high voltage cable accessories", IEEE Trans. Dielectr. Electr. Insul., Vol. 21, No. 1, pp. 5-15, 2014.

[8] I. A. Metwally, A.H. Al-Badi, A.S. Al-Hinai, F. Al Kamali and H. AlGhaithi, "Influence of design parameters and defects on electric field distributions inside mv cable joints". Proceedings of the $18^{\text {th }}$ Mediterranean Electro technical Conference MELECON, Limassol, Cyprus, 18-20 April 2016.

[9] D. D Chang, T.S. Sudarshan and J.E.Thompso "Analysis of electric stress distribution in cavities embedded within dielectric structures", IEEE Trans.On Elect.Insul.Vol, EI-21, No.2, pp. 213-219, 1986.

[10] A. A. Hossam-Eldin, S. S. Dessouky, S. M. El-Mekkawy, and R. A. AbdElAal "Analysis and simulation of field distribution in micro cavities in solid insulating materials", CEIDP 2007, Oct, Vancouver, Canda, pp792-796.

[11] S. S. Desouky, A. Z. El-Dein , R. A. Abd El-Aal , and N. A. A. ElRahman "A new contribution in reducing electric field distribution within/ around medium voltage underground cable terminations", Engineering, Technology \& Applied Science Research, Vol. 7, No. 5, 2017, 1963-1967

[12] COMSOL Multiphysics, AC/DC Module, available at: http://www. comsol.fi/products/acdc/

[13] A. Aarnio, "Characterization of non-metallic materials for medium voltage cable accessories", M.Sc. Thesis, Tampere: University of Technology, 2010. 
Faculty of Energy Engineering - Aswan University - Aswan - Egypt 